\title{
Evaluation of a Genus Specific rGroEL 1-524 IgM- ELISA and Commercially ELISA Kits During Course of Leptospirosis in Thailand
}

Santi Maneewatchararangsri ( $\nabla$ santi.man@mahidol.ac.th )

Mahidol University

Galayanee Doungchawee

Mahidol University Salaya campus

Thareerat Kalambaheti

Mahidol University

Viravarn Luvira

Mahidol University

Ngamphol Soonthornworasiri

Mahidol University

Pisut Vattanatham

Mahidol University

Urai Chaisri

Mahidol University

Poom Adisakwattana

Mahidol University

\section{Research Article}

Keywords: rGroEL1-524 IgM-ELISA, Leptospirosis, rural areas, seroprevalence surveys, reference tests

Posted Date: March 30th, 2021

DOl: https://doi.org/10.21203/rs.3.rs-352195/v1

License: (a) This work is licensed under a Creative Commons Attribution 4.0 International License. Read Full License 


\section{Abstract}

This work, we developed a genus-specific rGroEL ${ }_{1-524}$ IgM-ELISA assay for using as screening diagnosis of suspected leptospirosis among acute undifferentiated febrile illness patients during acute fever.

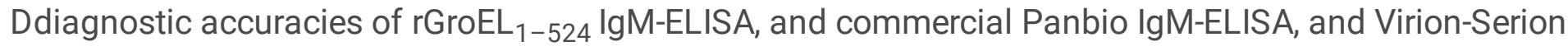
Classic IgG-ELISA were evaluated with Thai 107 leptospirosis sera, and 189 controls, compared to reference culture and/or MAT methods. Sensitivities were $91.7 \%, 59.6 \%$, and $17.7 \%$ for acute- infection, and were $97.1 \%, 54.8 \%$, and $9.7 \%$ for early detection at $1-3$ days post-onset of symptoms (DP01-3), and the specificities were $87.5 \%, 86.6 \%$, and $74.8 \%$ among controls, respectively. The rGroEL $1-524$ IgM-ELISA had high sensitivity, at $95.9 \%$ and $91.2 \%$ among culture and MAT negative cases. Impaired specificity on scrub typhus, possibly from antibody-cross reaction to ortholog GroEL. Commercial Panbio IgM-ELISA had sensitivities of $50 \%, 63.2 \%$, and $89.9 \%$ compared with culture, MAT-negative and single MAT-positive cases whereas Virion-Serion IgG-ELISA provided sensitivities of $13.3 \%, 10.5 \%$ and $71.4 \%$, respectively. A $\mathrm{rGroEL}_{1-524} \mathrm{IgM-ELISA}$ could be useful as a screening test for early diagnosis. The performance of the commercial ELISA suggests the applicability of IgM-ELISA for diagnosis, while IgG-ELISA is useful for seroprevalence surveys. However, confirmation by reference tests is recommended.

\section{Introduction}

Leptospirosis is a waterborne zoonosis with high incidence in tropical and sub-tropical areas, particularly rural areas and urban slum communities of developing and industrialized countries. Its epidemiology undergoes changes due to global warming and migration. The disease continues to be a global public health burden afflicting $0.1-1$ per 100,000 /population with a $10 \%$ case-fatality rate annually; it also has a socio-economic impact ${ }^{1}$. Epidemic outbreaks occasionally occur and are associated with flooding in epidemiological settings, such as Thailand, the Philippines, New Caledonia, Hawaii, and Nicaragua. The Thai Bureau of Epidemiology (BoE), Department of Disease Control, Ministry of Public Health, has reported incidence and case-fatality rates rate in 2019 of 3.26 and 0.04 per 100,000/population, with demographic shifts in Southern Ranong, Phang Nga, and Yala, northeastern Sisaket and Yasothon provinces, usually associated with occupations, such as agricultural farmers, laborers, students, government services staff, and also associated with recreational activities and travelers ${ }^{2-4}$. Disease is seasonal with a peak incidence in the late rainy season to early winter, and occasionally occurs after high rainfall and flooding, such as the epidemic outbreak in Loei, $2002^{5-6}$.

Humans are usually infected by pathogenic bacteria of the genus Leptospira which are allocated into three distinct phylogenetic clusters by the virulence of bacteria, into 13 pathogenic and 5 intermediately pathogenic species and free-living saprophytes ${ }^{7}$. The main disease-causing species in humans and other animals are pathogenic Leptospirainterrogans, L. borgpetersenii and L. kirschneri ${ }^{8}$; the intermediate group members that infect humans and mammals, causing mild disease, include L. broomii, L. fainei, L. inadai, $L$. licerasiae and $L$. wolffii, whereas non-pathogenic species do not cause disease ${ }^{7,9,10}$. Based on serology, the Leptospira spp. are classified into more than 300 different serovars and clustered into at least 24 
serogroups ${ }^{8,11}$. Human hosts commonly acquire infection by contact with bacterially contaminated urine, soil or water through abraded skin and mucous membranes, causing a wide range of clinical manifestations ranging from asymptomatic, though mild, and acute undifferentiated febrile illness $(\mathrm{AUFI})^{4}$ to severe leptospirosis such as Weil's disease, severe pulmonary hemorrhage syndrome, and aseptic meningitis, and potentially fatal illness ${ }^{12-13}$. Most patients present with non-specific febrile illness similar to other tropical diseases, such as dengue fever, rickettsioses, malaria, influenza, septicemic melioidosis, viral hemorrhagic fever, and enteric fever, making misdiagnosis possible ${ }^{14-16}$. Patients receiving early diagnosis and appropriate antibiotic therapies within 4-5 days post-onset of symptoms (DPO) have higher rates of recovery ${ }^{17-18}$. Delayed case diagnosis and late treatment can rapidly develop into severe complications where chemotherapy becomes useless ${ }^{2,16}$.

The World Health Organization recommends that a gold standard laboratory method for confirming leptospirosis diagnosis can be: (i) isolation of Leptospira by culture method, (ii) detection of organism DNA by PCR, and (iii) detection of antibody by microscopic agglutination test (MAT) ${ }^{17}$. MAT detects both immunoglobulin M (IgM) IgG agglutinating antibodies. However, MAT provides low sensitivity at early course of infection, as MAT can detect IgM antibodies after DPO 8 and requires testing paired-sera to confirm diagnosis. The test cannot differentiate current, recent, or past infections. Furthermore, MAT is technically demanding, time-consuming, and requires well-trained personnel for interpretation. Culture provides definite proof of leptospiral infection and could identify locally pathogenic serovars. However, culture is not useful as a diagnostic tool because by the time diagnosis is made by culture, antibodies are already detectable by serological techniques and the result is relatively delayed. MAT and culture methods have low diagnostic sensitivities but high specificity. Molecular diagnosis by PCR, quantitative PCR ( $q P C R)$, and recently whole genome sequencing, are not affordable in primary healthcare and in rural areas with resource-limited settings ${ }^{17,19}$. Hence, the development of reliable and valid diagnostic tests, providing high accuracy, is needed for the diagnosis of leptospirosis, so that the disease can be diagnosed and treated early in its course.

Immunodiagnostics, using the detection of IgM antibodies during acute illness, such as the enzymelinked immunosorbent assay (IgM-ELISA), immunofluorescence assay (IFA), and immunochromatography (ICT) formats ${ }^{20-21}$, have been implemented for the diagnosis of infectious diseases in the Tropics, such as leptospirosis, dengue fever, rickettsioses, and melioidosis ${ }^{22-24}$. IgM-ELISA is recommended by the World Health Organization (WHO) as useful in early diagnosis; the test has shown high sensitivity and specificity and is more sensitive than MAT. Commercially available ELISAs have shown inconsistent performance when evaluated in different epidemiological settings, and their accuracy needs systematic evaluation in Thailand ${ }^{17,18,25}$. In the present study, we evaluated two commercial ELISA tests, i.e., the IgM-ELISA (Panbio) and the IgG-ELISA (Virion-Serion) immunodiagnostic assays, for leptospirosis. The diagnostic accuracy of the commercial IgG-ELISA has been evaluated in limited studies and has not been evaluated in Thailand. The commercial Panbio IgM-ELISA has been evaluated in different settings, such as Malaysia ${ }^{26}$, Hawaii ${ }^{27}$, Laos $^{28}$, Southern Vietnam ${ }^{29}$, southern Sri Lanka ${ }^{30}$, and 
the French West Indies ${ }^{31}$. The Panbio IgM-ELISA demonstrated limited diagnostic sensitivity and specificity, at $76.1 \%$ and $82.6 \%$, respectively, when evaluated in high-prevalence northeast Thailand ${ }^{32}$.

Leptospira infections cause up-regulation of bacterial heat shock protein 60 (GroEL) in response to temperature-stress condition, eliciting long-lasting immune responses with high antibody titers. GroEL has shown to be a genus-specific immunodominant antigen as revealed by anti-Leptospira immune serum and leptospirosis paired-sera ${ }^{33-35}$. This protein has shown less cross-reactivity with sera from patients who have recovered from melioidosis or dengue hemorrhagic fever ${ }^{33-35}$. In this study, we developed an IgM-ELISA screening test using a genus-conserved region of $\mathrm{GroEL}_{1-524}$ (recombinant GroEL $_{1-524}$ ) as antigen to detect specific IgM antibody in blood specimens of suspected leptospirosis cases among AUFI patients and evaluated its diagnostic performance for early leptospirosis diagnosis using local Thai blood samples, compared to reference culture and MAT methods. We also assessed the usefulness of commercial ELISAs, i.e., Panbio Leptospira IgM-ELISA and the Virion-Serion classic Leptospira IgG-ELISA as screening tests for detecting anti-leptospiral antibodies using Thai blood samples in the context of disease outbreaks and compared to gold standard methods, including Leptospira isolation and MAT.

\section{Methods}

\section{Ethics and biosafety}

The protocol for using achieved sera and patient data was performed in concordance with the recommendation of the Declaration of Helsinki. Documentary Proof of Exemption Review was obtained from the Ethics Committee of the Faculty of Tropical Medicine, Mahidol University (MUTM-EXMPT2017005). Sample anonymity was maintained and all samples were re-coded without name and hospital ID. Biosafety was approved by the institute Biosafety Committee (MU2019-002).

\section{Study design}

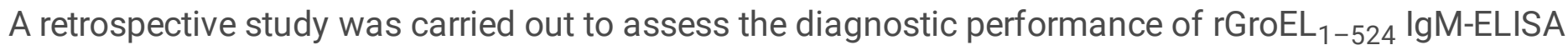
and to evaluate commercial whole-cell antigen-based ELISAs performance in detecting specific antibodies using local Thai blood samples compared with reference culture and MAT methods. The laboratory investigations were conducted at the Faculty of Tropical Medicine, Mahidol University, in Bangkok.

\section{Reference leptospirosis diagnosis}

Leptospira isolation was performed at the day of patient hospitalization by culturing blood specimens in EMJH (Ellinghausen-McCullough-Jonson-Harris) medium, and incubation for 16 weeks ${ }^{5}$. Sera were tested by MAT assay with 20 reference Leptospira serovars, as described previously ${ }^{5,36}$. Patients' specimens were investigated by cultivation and/or MAT. The reference diagnosis was conducted at Loei Provincial 
Hospital. Single leptospirosis sera were confirmed for MAT titers at the Faculty of Tropical Medicine, Mahidol University with reference strains recommended by the WHO. MAT-positive criteria were defined as single MAT titer of ${ }^{31}: 400$ in a single specimen, sero-conversion from negative to titer ${ }^{31}: 400$, or a fourfold rise in MAT titer using paired-sera. A MAT-negative sample was defined as MAT titer $£ 1: 50^{17,21,32,37-}$ 38.

\section{Leptospirosis patientsand sera}

A suspected leptospirosis case was clinically diagnosed based on WHO criteria, i.e., acute undifferentiated febrile illness (AUFI) patients (fever ${ }^{3} 38^{\circ} \mathrm{C}$ ) with headache, myalgia with history of exposure to animal reservoirs or flooded environments ${ }^{17}$. A confirmed leptospirosis case was defined as a clinically diagnosed, suspected leptospirosis case combined with positive laboratory diagnosis by culture method and/or MAT (single MAT titer ${ }^{31}: 400$ in single serum or 4-fold rise or seroconversion of paired-sera).

Confirmed leptospirosis sera $(n=133)$ were obtained from patients during an epidemic outbreak at Loei provincial hospital $(n=95)$, between July-October 2002, and sporadic cases at Nakhon Ratchasima and Sakhon Nakhon northeastern provinces $(n=38)$ before $2009^{5,36}$ (Fig. 1). The median time for onset of symptoms was 3 days (IQR: 1-10). Leptospirosis sera $(n=133)$ were acute sera $(n=52)$ collected on the first day of hospitalization during DP01-10 and classified into DP01-3 ( $n=31)$, and DPO 4-10 $(n=12)$, and convalescent sera $(n=45)$ collected 14 days later.

Among the sera, samples with a single MAT titer of 1:100-1:200 $(n=25)$ were excluded. All confirmed leptospirosis sera $(n=107)$ were classified according to reference tests into culture-positive samples $(n=30)$ and MAT-positive samples, including single MAT titer of ${ }^{31}: 400(n=28)$, and MAT-negative $(n=38)$ of seroconversion paired-sera. The most prevalent serogroups (serovars) among the MAT-positive sera were Autumnalis (Autumnalis, New), Australis (Australis, Bangkok, Bratislava), Icterohemorrhagiae (Copenhageni), Sarmin (Sarmin), and Sejroe (Sejroe) ${ }^{5}$.

\section{Control samples}

To assess the specificity of the ELISA tests, a panel of control samples (total no. $=210$ ) consisting of 60 non-endemic control plasma (seronegative and negative for leptospirosis IgM detection) and 150 laboratory-confirmed infectious diseases other than leptospirosis, were used. Control samples $(n=21)$ were excluded from this study because of insufficient sample volumes and a limited number of commercial test kits (Fig. 1). Non-endemic plasma controls were collected from healthy volunteers and undifferentiated febrile plasma $(n=60)$ at the hospital for Tropical Diseases, Bangkok, in 2014. Laboratory-confirmed other febrile illnesses were seropositive dengue paired-sera $(n=20)$, collected from undifferentiated fever; classic dengue fever patients at Sisaket Provincial Hospital, Srisaket Province, in 2013; scrub typhus samples $(n=20)$ were PCR positive, and sero-positive acute serum collected at Umphang Hospital, Tak Province, in 2018; influenza paired-sera $(n=20)$ were collected from 
hemagglutination inhibition seroconversion of $\mathrm{H} 1 \mathrm{~N} 1$-infected patients; malaria plasma $(\mathrm{n}=20)$ was collected from malaria vivax-positive patients in Tak Province $(n=20)$; and melioidosis sera $(n=20)$ were collected from IgM-seropositive melioidosis patients from northeastern Thailand, before 2018. Whole blood samples $(n=50)$ were collected from AUFI patients (total no. $=50$ ) admitted to the Hospital for Tropical Diseases, Bangkok, during the period 2013-2015. The samples were laboratory-confirmed murine typhus $(n=15)$, dengue $(n=30)$, and bacterial sepsis $(n=5)$ caused by E. coli, Streptococcus agalactiae, Salmonella Typhi, and Viridans Streptococci infections (Fig. 1) ${ }^{14}$. The samples were collected in microtubes and stored at $-70^{\circ} \mathrm{C}$.

\section{Production of a transformed $E$. coli carrying a recombinant GroEL ${ }_{1-524}-\mathrm{pET} 23 \mathrm{a}(+)$ plasmid.}

Briefly, DNA sequence encoding for $\mathrm{GroEL}_{1-524}$ was amplified by PCR reaction using specific primers GroEL-Ndel/F (5'-GGCCCATATGGCGAAAGATATTGAATAT-3') and GroEL-BamH/R (5'-TTGGATCC ATCTGGTTTGTCTGTGATTGT-3'). PCR reaction were performed in a volume of $25 \mathrm{~mL}$ of PCR super mix (Quantabio, MA, USA) containing $\mathrm{MgCl}_{2}$, dNTP, Taq DNA polymerase, each GroEL primer, and $100 \mathrm{ng}$ of genomic DNA extracted from $L$. interrogans serovar Icterohemorrhagiae. Amplifications were performed according to the following conditions: one cycle of $94{ }^{\circ} \mathrm{C}$ for $5 \mathrm{~min}, 29$ cycles of $94^{\circ} \mathrm{C}$ for $1 \mathrm{~min}, 55^{\circ} \mathrm{C}$ for $1 \mathrm{~min}$ and $72{ }^{\circ} \mathrm{C}$ for $1.30 \mathrm{~min}$, followed by a final period of $72{ }^{\circ} \mathrm{C}$ for $5 \mathrm{~min}$. The PCR product was analyzed under $1 \%$ agarose gel electrophoresis and visualized by Gel Documentation (Bio-Rad, California, USA).

The GroEL $L_{1-524}$ fragment was digested with $\mathrm{Ndel}$ and $\mathrm{BamHI}$ restriction endonucleases and ligated into a linearized plasmid backbone to produce a recombinant GroEL $_{1-524} \mathrm{pET} 23 \mathrm{a}(+)$ plasmid, subsequently introduced into BL21(DE3) E. coli. Positive transformed E. coli were PCR-screened using universal T7 primers. The GroEL ${ }_{1-524}$ sequence was verified by standard sequencing (Bioneer, Daejeon, Republic of Korea). The genetic map of the GroEL ${ }_{1-524} \mathrm{pET} 23 \mathrm{a}(+)$ plasmid is illustrated in supplementary Fig. S1.

\section{Production of recombinant $\mathrm{GroEL}_{1-524}$ protein.}

Recombinant $\mathrm{GroEL}_{1-524}$ protein was produced under $E$. coli expression system using a transformed $E$. coli strain bearing the recombinant $G r o E L_{1-524}$ plasmid. Briefly, E. coli was grown in Luria-Bertani broth containing $100 \mu \mathrm{g} / \mathrm{mL}$ ampicillin at $37^{\circ} \mathrm{C}$ with $200 \mathrm{rpm}$ shaking until the culture reached an $\mathrm{OD}_{600 \mathrm{~nm}}$ of 0.5. Thereafter, isopropyl $\beta$-d-1-thiogalactopyranoside (IPTG, $1 \mathrm{mM}$ ) was added to induce rGroEL $\mathrm{L}_{1-524}$ protein expression at $37^{\circ} \mathrm{C}$ with $200 \mathrm{rpm}$ shaking for $3 \mathrm{~h}$.

A soluble fraction from the IPTG-induced bacteria containing rGroEL $_{1-524}$ protein was prepared in phosphate-buffered saline (1×PBS, pH 7.4) using a French pressure cell press at 30 kilo-pounds persquare-inch, repeated 4 times. The rGroEL $_{1-524}$ protein was purified from the soluble proteins by native affinity chromatography using $\mathrm{Ni}^{2+}$-sepharose (GE Healthcare, Uppsala, Sweden). The purified $\mathrm{rGroEL}_{1-524}$ protein was concentrated in 1×PBS ( $\mathrm{pH}$ 7.4) using a 3-kDa cut-off Amicon Ultra filter (Merck Millipore, MA, 
USA) and was determined for protein concentration using a Bradford assay (Thermo Fisher Scientific, MA, USA). Aliquots of the protein $(1 \mathrm{mg} / \mathrm{mL})$ were lyophilized using the Labcono Freeze Dry system, and then kept at $-70^{\circ} \mathrm{C}$.

\section{SDS-PAGE and Western blotting}

Protein was analyzed under 13\% SDS-PAGE gel electrophoresis, denaturing condition, and Coomassie Brilliant Blue $\mathrm{G} 250$ stain. Antigenic specificity testing of the $\mathrm{GGroEL}_{1-524}$ protein was performed by probing the blotted membrane with anti-6xHis-Tag monoclonal antibody $(1: 1,000)$ (R\&D Systems, MN, USA) for 1 $\mathrm{h}$ at $25^{\circ} \mathrm{C}$, followed by HRP-conjugated goat anti-mouse IgG secondary antibody $(1: 2,000)$ (Jackson ImmunoResearch, PA, USA) for $1 \mathrm{~h}$ at $25^{\circ} \mathrm{C}$ (Southern Biotechnology, AL, USA). The reactive band was developed using 3,3-diaminobenzidine (DAB) chromogenic substrate (Thermo Fisher Scientific, MA, USA).

\section{In-house rGroEL $\mathrm{L}_{1-524}$ IgM-ELISA.}

Recombinant GroEL ${ }_{1-524}(1 \mathrm{mg})$ immobilized ELISA strips (Jet Biofil, Guangzhou, China) were prepared as follows: $\mathrm{GGroEL}_{1-524}$ protein in $100 \mu \mathrm{L}$ of carbonate-bicarbonate buffer ( $\mathrm{pH}$ 9.6) was immobilized on ELISA wells at $37^{\circ} \mathrm{C}$ for $24 \mathrm{~h}$ and the antigen-coated wells were washed using washing buffer (300 $\mu \mathrm{L} /$ well of PBST; $0.05 \%$ Tween 20 in $1 \times$ PBS, pH 7.4). Washing was conducted by an automated microplate washer (Tecan Trading AG, Switzerland) three times to remove unbound material. The coated wells were then incubated with blocking reagent ( $300 \mu \mathrm{L}$ of $1 \% \mathrm{BSA}$ in $1 \times \mathrm{PBS})$ for $1 \mathrm{~h}$ at $37^{\circ} \mathrm{C}$, followed by incubating the pre-blocked wells with $300 \mu \mathrm{L}$ of $2 \%$ sucrose solution at $25^{\circ} \mathrm{C}$ for $1 \mathrm{~h}$. The ELISA wells were washed after each incubation step, as described above, and then air-dried. The pre-blocked $\mathrm{rGroEL}_{1-}$ ${ }_{524}$ ELISA strips were packed with desiccant in press-seal bags and stored at $-20^{\circ} \mathrm{C}$ until use.

To detect anti-GroEL ${ }_{1-524} \operatorname{lgM}$ antibody, serum dilution $(1: 100,100 \mu \mathrm{L})$ in a serum diluent $(1 \times$ PBS containing $0.2 \%$ gelatin, $0.2 \%$ BSA), along with an internal positive control (pooled MAT-positive patient sera, where the adjusted actual optical density (AOD) exceeded 0.2) and a reagent control (serum diluent) were incubated in pre-blocked antigen-coated wells at $37^{\circ} \mathrm{C}$ for $1 \mathrm{~h}$, followed by washing three times with PBST. Thereafter, HRP-conjugated goat anti-human IgM antibody (100 mL, 1:2,000) (Southern Biotechnology, AL, USA) was added to ELISA wells at $37^{\circ} \mathrm{C}$ for $1 \mathrm{~h}$ incubation. ABTS chromophore diammonium salt (EMD Millipore, Germany) substrate solution $(1 \mathrm{mg} / \mathrm{mL}$ ABTS tablet in $0.1 \mathrm{M}$ sodium citrate buffer) was added $(100 \mu \mathrm{l})$, and the plate was incubated for $15 \mathrm{~min}$ at $37^{\circ} \mathrm{C}$, after which $100 \mu \mathrm{L}$ of $1 \%$ SDS solution was added to stop reaction. The optical density (OD) was measured at wavelength 410 $\mathrm{nm}$ against the reference $650 \mathrm{~nm}\left(\mathrm{OD}_{410 \mathrm{~nm} / 650 \mathrm{~nm})}\right)$ using a microplate reader (Bio-Tek Instruments, VT, USA). Sample AOD was calculated by subtracting the OD of the reagent blank. The IgM-ELISA assay is valid when the OD of the reagent blank is $<0.2$ and the positive AOD control is ${ }^{3} 0.2$. A rGroEL ${ }_{1-524}$ IgMELISA protocol was optimized and the optimal concentration of $\mathrm{rGroEL}_{1-524}$ was $1 \mu \mathrm{g} /$ well; serum dilution was 1:100 and secondary antibody dilution was 1:1,000-1:3,000 dilutions.

Panbio Leptospira IgM-ELISA. 
The diagnostic performance of the commercial Panbio Leptospira IgM-ELISA (Abbott Diagnostics, Illinois, USA) (Lot no. 02P10E001), using Leptospira genus-specific antigen, was assessed in Thai blood samples. The Panbio IgM-ELISA protocol was performed as per the manufacturer's instructions,

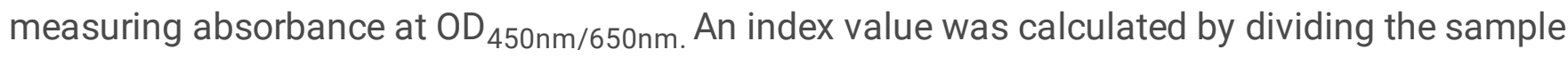
absorbance by the cut-off value. The result was expressed as Panbio units (index value multiplied by 10). Interpretation of the validity results was as follows: Panbio units (anti-Leptospira IgM) of $<9$ was a negative result, suggesting no evidence of recent infection, Panbio units ${ }^{3} 9$ to $<11$ was an equivocal result, suggesting possible recent infection, and Panbio units ${ }^{3} 11$ was positive by IgM detection and interpreted as a recent or current infection. An equivocal result was considered a positive result. The Panbio IgM-ELISA test performance showed $96.5 \%$ sensitivity and $98.5 \%$ specificity and has been validated to detect leptospira infections by serovars Pomona, Copenhageni, Australis, Canicola, Grippotyphosa, Tarsassovi, Hardjo, Madanesis, Kremastos, Nokolaevo, Cellodoni, Szwajizak, and Djasiman.

\section{Virion-Serion Classic Leptospira IgG-ELISA.}

Institute Virion-Serion ELISA Classic Leptospira IgG (Institut Virion/Serion GmbH, Warburg, Germany) (order no. ESR $125 \mathrm{G}$ ) was used to detect anti-Leptospira IgG, IgA, or IgM antibodies from serum or plasma using a crude membrane extract of L. biflexa serovar Patoc strain Patoc I, which contains genusspecific epitopes for all Leptospira spp. Virion-Serion IgG-ELISA procedure was performed as per the

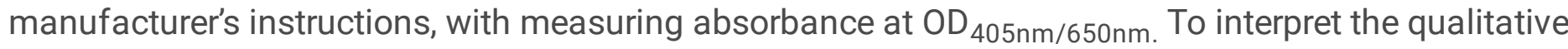
results, the upper and lower cut-off range was calculated according to parameters provided with the kit. Actual OD (AOD) value (anti-Leptospira IgG) lower than the cut-off was a negative result suggesting no evidence of past exposure, an AOD value in the cut-off range was a borderline result, suggesting possible past exposure, and an AOD value higher than the upper cut-off was positive by IgG detection, suggesting previous exposure. Borderline was considered a positive result. The diagnostic performance of the VirionSerion Leptospira IgG-ELISA was $96.7 \%$ sensitivity and $99.8 \%$ specificity.

\section{Evaluation of diagnostic accuracy.}

The Standards for Reporting of Diagnostic Accuracy studies (STARD 2015) checklist for reporting diagnostic accuracy is provided in supplementary Table $\mathbf{S 6}$.

Sample size was estimated as a minimum of 35 cases and control samples to achieve $90 \%$ sensitivity and specificity at $95 \%$ confidence interval $(\mathrm{Cl})$ and $7 \%$ precision. All sera were tested as anonymous samples. A total of 107 leptospirosis sera and 189 controls (Fig. 1) were randomly selected to evaluate the diagnostic performance of the following tests: (i) $\mathrm{rGroEL}_{1-524}$ IgM-ELISA, (ii) commercial Panbio Leptospira IgM-ELISA, and (iii) Virion-Serion Classic IgG-ELISA. The estimated diagnostic sensitivity and specificity with $95 \% \mathrm{Cl}$ were calculated by 2 ' 2 cross-tabulation table. The hook effect (prozone effect) was determined in serial dilutions of 1:100 and 1:1,000 in MAT-positive leptospirosis sera and other febrile-illness patient sera. 


\section{Bioinformatics.}

Conservation of GroEL sequences of the selected in the genus Leptospira and among GroEL orthologs were determined using Clustal Omega multiple sequence alignment program interface ${ }^{39}$ and the results were analyzed using the BioEdit sequence alignment editor tool. Linear B-cell epitopes of the $L$. interrogans serovar Icterohemorrhagiae GroEL sequence were computationally predicted using a Bepipred-1.0 Linear Epitope Prediction tool ${ }^{40}$.

\section{Statistical calculations.}

Data were collected in Microsoft Excel and were analyzed using MedCalc Statistical Software version 19.2.5 (MedCalc Software Ltd, Ostend, Belgium; https://www.medcalc.org; 2020). Cut-off value was determined using receiver operating characteristic (ROC) curve analysis using paired leptospirosis sera and non-endemic control and other febrile-illness control. Diagnostic parameters were calculated as follows: sensitivity $=[$ (true positive $(T P) /(T P+$ false negative $(F N))] \times 100 ;$ specificity $=[($ true negative $(\mathrm{TN})) /(\mathrm{TN}+$ false positive $(\mathrm{FP}))] \times 100$. Normal distribution was tested using the Kolmogorov-Smirnov test. The Mann-Whitney test was used in non-normal distributed data. $p<0.05$ was considered statistically significant.

\section{Results}

\section{Verification of $\mathrm{GroEL}_{1-524}$ sequence conservation within the genus Leptospira spp. and among orthologous GroELproteins.}

The Leptospira GroEL $\mathrm{L}_{1-524}$ sequence had a high degree of homology, at $99 \%$ (522/524), sequence identity to those of other leptospiral serovars in the Leptospira spp. To evaluate their protein-sequence conservation across genera, GroEL orthologs among other tropical infectious diseases, i.e., leptospirosis, scrub typhus, melioidosis, and malaria, were studied. Orthologous GroEL proteins of influenza A and dengue viruses were not found from the NCBI database. The orthologous GroEL proteins of $L$. interrogans,B. pseudomallei, P. vivax, and $O$. tsutsugamushi organisms demonstrated $60.5 \%, 51.3 \%$, and $51.9 \%$ identity, respectively, compared with the cloned Leptospira GroEL $_{1-524}$ sequence (Fig. 2). The GroEL $_{1-524}$ sequence was highly conserved in the genus Leptospira spp. and shared conserved peptides among orthologous GroEL proteins.

\section{Prediction of GroEL $_{1-524}$ linear B-epitope peptides.}

Figure 2 illustrates the 15 predicted linear B-epitope peptides distributed in the $\mathrm{GroEL}_{1-524}$ sequence. Two peptides of 30-LGPKGRN-36 (85.7\% identity) and 404-AAVEEGIVPG-413 (100\% identity) have been shown to be highly conserved among 4 pathogens. The epitope peptide similarity suggests a degree of immunological cross-reactivity among leptospirosis, scrub typhus, malaria, and melioidosis sera.

\section{Recombinant GroEL $_{1-524}$ protein preparation.}


The C-terminal His-Tagged $\mathrm{GroEL}_{1-524}$ protein was produced as a soluble protein of estimated $58.7 \mathrm{kDa}$ (supplementary Fig. S1). The purified $\mathrm{rGroEL}_{1-524}$ protein was verified for antigenic specificity, which revealed a reactive band at $60 \mathrm{kDa}$. The $\mathrm{rGroEL}_{1-524}$ protein was used as antigen in the development of a recombinant antigen-based IgM-ELISA.

\section{IgM sera reactivity of leptospirosis and controls to GroEL $_{1-524}$ antigen by IgM-ELISA.}

The IgM antibody reactivities of leptospirosis paired-sera in an optimized $\mathrm{rGroEL}_{1-524} \operatorname{lgM}$-ELISA were presented as AOD values with a range of 0-1.01. Median AOD values were 0.40 , and 0.43 for leptospirosis paired-sera, and were significantly higher than the non-endemic control $(p<0.0001)$ and other febrile illness $(p<0.001)$. The IgM reactivities of leptospirosis paired-sera versus scrub typhus subgroup were not significantly different ( $p=0.15, p=0.09$, respectively) (Table 1, Fig. 3 ). The sera reactivities among culture and MAT-positive subgroups were not significantly higher than the controls. The median IgM reactivities of single MAT-positive ( $\left.{ }^{3} 1: 400\right)$ and MAT-negative seroconversion were 0.50 and 0.45 for acute-sera, and 0.43 and 0.24 for convalescent sera, respectively (Fig. 3B, supplementary Table S1).

\section{Determination of optimal cut-off values.}

To optimize cut-off values, ROC curves were generated from leptospirosis paired- sera and controls to define the optimal OD and was defined as 0.15 OD ELISA to achieve an estimated sensitivity of $91.6 \%$ and $95.5 \%$ for the paired-sera, and a specificity of $87.5 \%$ for the controls.

The results of IgM detection by Panbio IgM-ELISA and IgG detection by Virion-Serion IgG-ELISA on leptospirosis paired sera and controls are illustrated with adjusted cut-off values in supplementary Fig. S2. The optimal threshold for Panbio IgM-ELISA cut-off value was ${ }^{3} 7$ Panbio units and the optimized cutoff value for Serion IgG-ELISA was ${ }^{3} 0.35$ OD ELISA to achieve higher sensitivities.

\section{Diagnostic accuracy of rGroEL ${ }_{1-524}$ IgM-ELISA.}

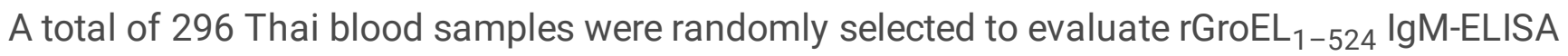
performance compared with culture and MAT methods. The cut-off AOD value was taken as 0.15 . The diagnostic sensitivities of $\mathrm{rGroEL}_{1-524}$ IgM-ELISA were $91.7 \%$ for acute sera and $95.6 \%$ for convalescentsera (Table 1). Among the leptospirosis sera (Fig. 3), the in-house IgM-ELISA demonstrated sensitivities of 95.9\% in culture-positive, $91.2 \%$ in MAT-negative of seroconversion, and $88.2 \%$ in single MAT-positive subgroups.

The specificity was $87.5 \%$ for the controls, $92.5 \%$ for the non-endemic control, $72.2 \%$ and $100 \%$ among other febrile-illness controls (Table 1) with corresponding AUC values of $0.93(p<0.001), 0.83(p=0.02)$, and 0.99 ( $p<0.001)$, respectively. Of the following febrile illness specificities, $60.0 \%$ for dengue fever, $54.5 \%$ for scrub typhus, $81.8 \%$ for influenza, $70.0 \%$ for malaria, and $91.7 \%$ for melioidosis, and $100 \%$ for bacteremia, murine typhus, or dengue fever (supplementary Table S1). Notably, no dilution effect on the non-leptospirosis control sera across the serial dilutions of 1:100-1:1,000 was found. 


\section{Evaluation of diagnostic accuracy of GroEL $_{1-524}$ IgM-ELISA during course of leptospirosis using culture and MAT as reference standard.}

The diagnostic sensitivities on DPO1-3, and DPO4-10 of acute-phase and DPO 14 (convalescent sera)

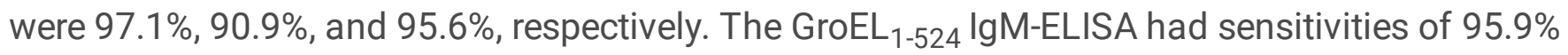
compared to culture method, and $91.2 \%$ compared to MAT-negative seroconversion criteria as early as DP01-3. Sensitivity was slightly decreased to $88.2 \%$ on MAT $^{3} 1: 400$ (Table 1 ).

\section{Diagnostic performance of commercial ELISA test kits against local Thai sera.}

The performance of the commercial ELISAs was determined using 296 samples of leptospirosis paired sera and controls with manufacturer's cut-offs. The results (supplementary Table S2) showed poor sensitivities, with $44.2 \%$ for IgM-detection and $7.8 \% \operatorname{lgG}$ detection for acute-phase. The diagnostic sensitivities of the Panbio IgM-ELISA were re-calculated with adjusted cut-offs and the performance was increased to $59.6 \%$ and $71.4 \%$, whereas the IgG-ELISA showed $17.7 \%$, and $48.9 \%$ sensitivities for leptospirosis paired-sera, respectively. The sensitivities of the commercial ELISAs were $54.8 \%$ (Panbio IgM-ELISA), and $9.7 \%$ (Virion-Serion IgG ELISA) for early detection on DPO1-3, and $50 \%$ and $13.3 \%$ for culture-positive samples, and were $63.2 \%$, and $10.5 \%$ for MAT-negative samples by seroconversion criteria. Sensitivities of commercial ELISAs were increased to $89.9 \%$ (Panbio) and $71.4 \%$ (Serion) on single MAT ${ }^{3} 1: 400$ samples (Table 2).

\section{Analysis of anti-rGroEL $\mathrm{L}_{1-524}$ IgM-negative leptospirosis sera.}

False negative results were investigated primarily for seronegative (MAT<1:50) acute samples on DP01-3 $(n=4)$ and convalescent sera $(n=2)$; they had IgM reactivities at 1:100 dilution of $0.06-0.13$. Anti-rGroEL 1 ${ }_{524}$ negative samples on culture positive samples may have arisen from window period of active infection. False-negative of seronegative (MAT£1:50) and/or anti-Leptospira Panbio IgM positive detection suggested insufficient GroEL antigen-specific IgM antibody, or under the limit of detection by IgM-ELISA. False negatives arising from the prozone phenomenon were not observed in the MAT-positive serum diluted 1:1,000. The hook effect on the $\mathrm{MAT}^{3} 1: 400$ sera was seen with one convalescence sample (MAT=1:3,200) (supplementary Table S3).

\section{Analysis of false positive samples among controls by $\mathrm{rGroEL}_{1-524}$ IgM-ELISA.}

IgM positivity among controls by $\mathrm{rGroEL}_{1-524}$ IgM-ELISA was $7.5 \%$ (supplementary Table S4). All false positive samples were negative for anti-Leptospira IgM- and IgG- detections by commercial ELISAs, suggesting the false positives in the non-endemic control may have arisen from non-specific binding or pre-existing antibodies (background antibody) in those samples. Analysis of false-positive samples among the other febrile illness control is summarized in supplementary Table S4. 
Melioidosis samples had 8.3\% (1/12) false-positive results by rGroEL ${ }_{1-524}$ IgM-ELISA. Serum had antiLeptospira IgG positivity by commercial test, which suggested antibody cross-reactivity from previous exposure. The scrub typhus sera showed IgM reactivity ranging from $0.03-1.07 ; 45.5 \%(5 / 11)$ were deemed false positives. Two of the false positives had anti-Leptospira IgM positivity, which suggested antibody cross-reactivity from recent or current exposure or leptospirosis-scrub typhus co-infection. Three of the false-positive results were likely caused by antigen cross-reactivity to ortholog GroEL antigen (Fig. 2) or pre-existing antibody from endemic leptospirosis.

Two of 3 false-positive malaria samples had anti-Leptospira IgG positivity by Virion-Serion IgG-ELISA detection. The false-positive reactions may be related to pre-existing antibodies from previous exposure. One malaria sample had false-positive results by a in-house IgM-ELISA, which suggested antigen crossreactivity to ortholog GroEL antigen or preexisting antibody.

Two of ten influenza samples had false-positive results by IgM-ELISA (AOD, 0.16-0.2) at 1:100 dilution, but all were negatives for anti-Leptospira IgM and IgG detections by the commercial tests. False positives were likely caused by non-specific reactions. False positives of dengue samples were 40\% (4/10) by inhouse IgM-ELISA. Three of the false-positive samples had negative for anti-Leptospira detection, which suggested false positive may arise from endemic background antibody. One false-positive dengue sample had anti-Leptospira IgM positivity, which suggested recent or current infection and leptospirosisdengue coinfection.

\section{Discussion}

A genus-specific antigen-based ELISA using immunodominant outer membrane as antigens, such as LipL32, LipL41, Loa22, LigA, Lsa63, and GroEL, and in combinations (multiple antigens), have been widely developed for use as a screening test for leptospirosis $35,36,41-42$. This study developed a prototype IgM-ELISA using a recombinant GroEL $_{1-524}$ formatted antigen for an early laboratory screening test for leptospirosis and evaluated its diagnostic accuracy in the context of disease outbreaks in the Thailand setting, compared with reference standard methods. We produced recombinant $\mathrm{GroEL}_{1-524}$ protein (Cterminal deletion of 22 amino acids) and used as ELISA antigen to detect anti-rGroEL ${ }_{1-524} \operatorname{lgM}_{\text {antibody }}$ during course of illness for early diagnosis of suspected cases among AUFI caused by other infections. The heat shock GroEL chaperonin has shown potential as a diagnostic marker in leptospirosis based on its upregulated expression during infection (temperature upshift). GroEL has been shown to be an immunodominant antigen and has shown less cross-reactivity with melioidosis and dengue hemorrhagic fever $^{33-35}$. The immunoreactivity of severe leptospirosis, such as pulmonary involvement and renal failure, to recombinant GroEL has been shown to have $90.6 \%$ sensitivity and $94.9 \%$ specificity ${ }^{43}$. In addition, $\mathrm{GroEL}_{1-524}$ sequence was highly conserved within the genus Leptospira and shared lower sequence conservation with the orthologous GroEL (Fig. 2). We evaluated the diagnostic performance of the $\mathrm{rGroEL}_{1-524}$ IgM-ELISA using leptospirosis paired-sera derived from northeastern Thailand, i.e., Loei, Nakhon Ratchasima, Sakol Nakhon, and controls of healthy volunteers and AUFI patients from non- 
endemic Bangkok and other febrile illnesses, compared to MAT and culture reference methods. Leptospirosis sera from Loei were collected from an outbreak in 2002. Samples were positive by Leptospira isolation (30 culture-positive acute sera) and by seroconversion criteria. A small sample size of 28 single MAT ${ }^{3} 1: 400$ leptospirosis sera was obtained.

In the present study, a single IgM-ELISA was designed as a highly sensitive screening test. Cut-off was determined to be 0.15 AOD for single IgM-ELISA testing to achieve an estimated sensitivity of $91.6 \%$ and $95.5 \%$ for paired-sera and specificity of $92.5 \%$, and $76.0 \%$ among the non-endemic and febrile controls (supplementary Table S5). One limitation of acute-phase lgM testing with a single specimen is that people in endemic areas are expected to have pre-existing antibodies causing impaired specificity. ELISA results give no indication of the infecting serovar, and a confirmatory diagnosis of leptospirosis should be performed. As IgM antibody usually persists for 5 months ${ }^{44}$, ELISA can be used as a simple and rapid laboratory screening test for the diagnosis of leptospirosis for several months after onset of symptoms.

The sensitivities of the $\mathrm{rGroEL}_{1-524} \operatorname{lgM}$-ELISA were $91.7 \%$ and $95.6 \%$ for leptospirosis paired sera, and the specificity was $87.5 \%$ among the controls. Lessa-Aquino et al. ${ }^{35}$ reported GroEL IgM-ELISA sensitivities of $90 \%$ and $92.0 \%$ and specificities of $53.8 \%$ and $62.5 \%$ in paired sera. A systematic review and metaanalysis of the performance of Leptospira IgM-ELISA has averaged $84 \%$ sensitivity and $91 \%$ specificity for acute infection ${ }^{45}$. The GroEL $_{1-524} \mathrm{IgM}_{\mathrm{gMLISA}}$ had higher diagnostic performance than previous reports ${ }^{35,45}$ due to the refined GGroEL $_{1-524}$ molecule being able to encompass more antigenic moieties of the whole genus. The prototype IgM-ELISA had high sensitivities of $95.9 \%$ in culture-positive sera, $91.2 \%$ in seroconversion samples, and $88.2 \%$ in MAT-positive subgroups. With cut-off of 0.2 AOD, expected test performance was $87.5 \%$ and $86.7 \%$ for sensitivity in paired-sera and $81.5 \%$ specificity in the febrile control (supplementary Table S5). The most prevalent serovar infecting patients in the sera used in this study were Bratislava, Autumnalis, Australis, New, Sarmin, and Bangkok ${ }^{5}$, while Autumnalis, Bratislava, and Pyrogenes were the most common serovars in Thailand in 2003-2012 ${ }^{46}$. The in-house IgM-ELISA can detect IgM antibody early as DP01. The false-negative results on acute-phase by in-house IgM-ELISA may be due to the long window period by the dynamics of antibody production. Symptomatic patients may have no antibody or low levels during 1-2 weeks post-exposure and the antibody titer will rise with time. We found 2 false negative convalescent sera, may cause by delayed response which sometimes occur over 30 days after infection ${ }^{20}$.

Leptospirosis infections are often under-reported due to false-negatives among mild cases or have already received antibiotics, suppressed immunity, or in the very early or late phase of the immune response. Co-infection patients, weak or cross-reaction may occur. The false-positive rate among febrile

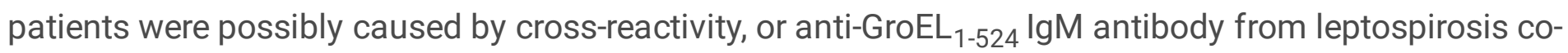
infections, and pre-existing lgM antibody from patients with recent exposure in endemic areas.

Commercial ELISAs have been used for the diagnosis of leptospirosis in Thai endemic settings, such as the Panbio Leptospira IgM-ELISA, and Virion-Serion classic Leptospira IgM/lgG. The performances of 
commercial ELISA tests vary with geographical settings, with the sensitivity of the Leptospira IgM-ELISA being $35-76 \%$ and specificity $76-98 \%$ in different endemic settings ${ }^{27-32,45}$. These ELISA tests use wholecell lysates from pathogenic L.interrogans, intermediate $L$. fainei, or saprophytic $L$. biflexa antigens to detect genus-specific anti-Leptospira lgM/IgG antibodies. Heterogeneous native antigens in ELISA tests may not recognize the local serovars, so their sensitivities are frequently poor and has been limited by the heterogeneity of host immunological responses to native antigens.

Whole-cell based ELISAs (Panbio IgM- and Virion-Serion IgG-ELISA) demonstrated poor sensitivity against local Thai leptospirosis paired sera (Table 2, supplementary Table S2). Another study showed that the Panbio IgM-ELISA yielded $90.8 \%$ positivity among samples from northeastern Thailand ${ }^{32}$. IgM antibodies appear earlier than IgG antibodies and remain detectable at low titers for months or even years. An IgG titer of 1:100 can be present due to past infection. Poor sensitivity of whole-cell based ELISA, which was affected by serogroup-specific antigen or whole-cell antigen, may not recognize local infecting strains in different endemic areas ${ }^{20,32,47}$. IgM-dominant and IgG-dominant $L$. biflexa serovar Patoc antigen, i.e., LPS, cytoplasmic, secreted and envelop membrane proteins, did not encompass local infecting serovars in the genus Leptospira. L. biflexa Patoc I antigen is known to cross-react with several serovars, but usually does not cross-react with animal strains. The most predominant infecting serovars in suspected patients from 2003-2004 in Thailand were serovars Autumnalis, Bataviae, Pyrogenes, Javanica, Hebdomadis and Grippotyphosa ${ }^{48}$. The most predominant infecting serovars between 2010-2015 were serovars (associated reservoir) Shermani (cattle, buffalo, pig), Bratislava (livestock, i.e., cattle, buffalo), Panama, and Sejroe (rodents) ${ }^{49}$. Another study examined the potential risk of a leptospirosis outbreak in Bangkok and Nakhon Pathom, between 2011-2012, and found L. wolffii and intermediate L. liceraseae ${ }^{50}$. Poor sensitivity can be attributed to several factors, such as acute serum being collected too early in the course of illness (less than DP04-5), inadequate IgM antibody level in the patient, second or subsequently episode of infection, leading to IgG antibody production, and patient receiving antibiotic medication. To improve specificity due to a high background antibody among the seropositive population requires validation and adjustment of the cut-off. In this study, we optimized cut-offs for commercial ELISAs. An adjusted Panbio unit of 37 showed a sensitivity improvement to $54.8 \%$ on DP01-3 acute-phase, and specificity of $86.6 \%$. The Panbio IgM-ELISA provided sensitivity and the ability to detect IgM antibodies early as DP01-3. Virion-Serion IgG-ELISA with an adjusted cut-off provided $17.7 \%$ and $48.9 \%$ sensitivity on leptospirosis paired-sera, and $81.7 \%$ specificity among the controls. False-positive IgG detection showed $19 \%$ among the controls ( $10 \%$ for non-endemic samples, $25 \%$ for each infection, i.e., dengue, malaria, scrub typhus, and melioidosis). IgG seropositivity rates of $17.7 \%$ and $48.9 \%$ on paired sera suggested IgG responses should be due to epidemic leptospirosis in Loei rather than background antibody, with $10 \%$ seropositivity among healthy and AUFI patients in low-prevalent leptospirosis areas, such as Bangkok.

Several studies have reported that ELISA-based assays detect anti-Leptospira IgM antibodies earlier than MAT assay during the early course of disease ${ }^{20,38,51}$. Nicofa et al. ${ }^{20}$ suggested that Leptospira-specific IgM antibodies appear 1-2 days earlier than the agglutinating antibodies detected in the MAT assay; 
therefore, earlier positive results could be expected from our genus-specific IgM detection. We found that the sensitivities in seronegative and culture-positive acute-sera were $91.2 \%$, and $95.9 \%$ for a prototype IgM-ELISA, and $63.2 \%$ and $50.0 \%$ for commercial IgM-ELISA, respectively. An unvalidated diagnostic test with poor specificity may contribute to over-diagnosis of leptospirosis, because IgM antibodies from past infections are frequently detected among people living in endemic areas ${ }^{20,51}$.

The cross-reactivity of the GroEL $_{1-524}$ IgM-ELISA was evaluated using sera from the local population, and on a non-leptospirosis febrile control group. The specificity of anti-Leptospira IgM detection is limited in pathogens expressing orthologous GroEL proteins, such as scrub typhus, malaria, and melioidosis,

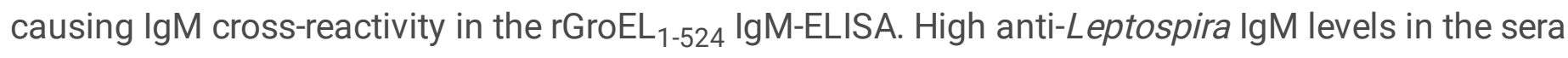
collected from patients along the Thai-Myanmar border have been reported ${ }^{52,53}$. However, it should be noted that cross-reactivity with bacterial infections can occur when patients harbor co-infections or have cross-reactive antibodies, especially in the early phase of leptospirosis when the IgM-ELISA lacks full specificity ${ }^{20}$.

The varied sensitivities likely reflect different case definitions and control groups, timing of collection, local prevalent serovar distribution, and the platform and protocol used in detection. A significant limitation of the rGroEL $_{1-524}$ IgM-ELISA was poor specificity for leptospirosis-endemic areas. The test specificity was affected by ortholog GroEL antigen cross-reactivity, antibody cross-reactivities caused by previous exposure, and co-infections. Co-infections with leptospirosis were not assessed in samples from other febrile illnesses. The use of the $\mathrm{rGroEL}_{1-524} \mathrm{IgM}$-ELISA as a screening test for leptospiral infection would facilitate the difficult reference and differential tests. However, the test should not be used as the sole criterion for diagnosing leptospirosis. The ELISA results must be confirmed by convalescent serum. MAT is still recommended for disease confirmation and epidemiological study, and Leptospira isolation and molecular characterization should be performed for confirmation of the infecting serovars ${ }^{54}$.

\section{Conclusion}

Our data demonstrated that IgM-ELISA using rGroEL $_{1-524}$ antigen has sufficiently high sensitivity to

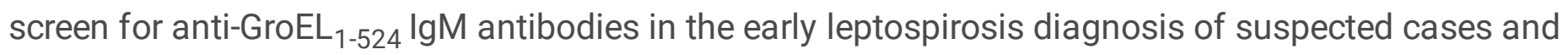
among high-risk groups during leptospirosis epidemics. However, diagnostic specificity needs to be improved for implementation in areas highly prevalent with infectious tropical diseases. The commercial ELISA performance data suggest the applicability of IgM-ELISA for early diagnosis during disease outbreaks in low-prevalence areas for leptospirosis. IgG-ELISA is useful for seroprevalence surveys, however, confirmation by reference tests is recommended.

\section{Declarations}

\section{Author information}


Department of Molecular Tropical Medicine and Genetics, Faculty of Tropical Medicine, Mahidol University, Bangkok, Thailand

Santi Maneewatchararangsri, Pisut Vattanatham

Center for Research and Innovation, Faculty of Medical Technology, Mahidol University Salaya campus, Nakhon Pathom, Thailand

Galayanee Doungchawee

Department of Microbiology and Immunology, Faculty of Tropical Medicine, Mahidol University, Bangkok, Thailand

Thareerat Kalambaheti

Department of Clinical Tropical Medicine, Faculty of Tropical Medicine, Mahidol University, Bangkok, Thailand

Viravarn Luvira

Department of Tropical Hygiene, Faculty of Tropical Medicine, Mahidol University, Bangkok, Thailand Ngamphol Soonthornworasiri

Department of Tropical Pathology, Faculty of Tropical Medicine, Mahidol University, Bangkok, Thailand Urai Chaisri

Department of Helminthology, Faculty of Tropical Medicine, Mahidol University, Bangkok, Thailand

Poom Adisakwattana

Data availability

The datasets used and analyzed from the current study are available from the corresponding author on reasonable request.

\section{Acknowledgements}

We greatly appreciate Prof. Dr. Pilaipan Puthavatana of the Faculty of Medical Technology, Mahidol University, for providing influenza sera; Assoc. Prof. Dr. Yupaporn Wattanagoon, Faculty of Tropical Medicine, Mahidol University, who provided scrub typhus sera; Assoc. Prof. Dr. Narisara Chantratita, Faculty of Tropical Medicine, Mahidol University, who provided melioidosis sera; Assoc. Prof. Dr. Pongrama Ramasoota, Centre of Excellence for Antibody Research, and Assoc. Prof. Dr. Kriengsak Limkittikul, Department of Tropical Pediatrics, Faculty of Tropical Medicine, who provided dengue sera; Prof. Dr. Jetsumon Prachumsri, Mahidol Vivax Research Unit, Faculty of Tropical Medicine, who provided 
vivax malaria sera; and Dr. Rapatbhorn Patrapuvich, Drug Research Unit for Malaria, Faculty of Tropical Medicine, who provided healthy control sera and AUFI sera.

We thank Edanz Group (https://en-author-services.edanzgroup.com/) for editing a draft of this manuscript.

\section{Funding}

This work was supported by the Center of Excellence in Medical biotechnology, Thailand and the Faculty of Tropical Medicine, Mahidol University.

\section{Author contributions}

S.M. contributed to the study conception and design. Material preparation, laboratory work and data collection were performed by all authors. Laboratory and statistical analysis were performed by S.M., P.V., and N.S. Manuscript was mainly written by S.M. and all authors commented on the manuscript. All authors read and approved the final manuscript.

\section{Competing interests}

The authors declare no competing interests.

\section{References}

1. Abela-Ridder, B., Sikkema, R., \& Hartskeerl, R. Estimating the burden of human leptospirosis. Int. J. Antimicrob. Agents. 36 (Suppl 1), S5-7 (2010).

2. Bharti, A.R. et al. Leptospirosis: a zoonotic disease of global important. Lancet. Infect. Dis. 3(12), 757-771 (2003).

3. Bandara, M., Ananda, M., Wickramage, K., Berger, E., \& Agampodi, S. Globalization of leptospirosis through travel and migration. Global. Health. 10, 61 (2014).

4. Niwattayakul, K., Homvijitkul, J., Niwattayakul, S., Khow, O., \& Sitprija, V. Hypotention, renal failure, and pulmonary complications on leptospirosis. Fail. 24(3), 297-305 (2002).

5. Niwetpathomwat, A., Niwatayakul, K., \& Doungchawee, Surveillance of leptospirosis after flooding at Loei Province, Thailand by year 2002. Southeast Asian. J. Trop. Med. Public. Health. 36(Suppl 4), 202-205 (2005).

6. Suwanpakdee, S. et al. Spatio-temporal patterns of leptospirosis in Thailand: is flooding a risk factor?. Epidemiol. Infect. 143(10), 2106-2115 (2015).

7. Vincent, A.T. et al. Revisiting the taxonomy and evolution of pathogenicity of the genus Leptospira through the prism of genomics. PLos Negl. Trop. Dis. 13(5), e0007270 (2019).

8. Picardeau, M. Virulence of the zoonotic agent of leptospirosis: still terra incognita? Nat. Microbiol. 15(5), 297-307 (2017). 
9. Adler, B., \& de la Pena-Moctezuma, A. Leptospira and leptospirosis. Vet. Microbiol. 140, 287-296 (2010).

10. Ko, A., Goarant, C., \& Picadeau, M. Leptospira: the dawn of the molecular genetics' era for an emerging zoonotic pathogen. Rev. Microbiol. 7(10), 736-747 (2009).

11. Thibeaux, R. et al. Deciphering the unexplored Leptospira diversity from soils uncovers genomic evolution to virulence. Genom. 4(1), e000144 (2018).

12. Ganoza, C.A. et al. Asymtomatic renal colonization of humans in the Peeruvian Amazon by Leptospira. PLos Negl. Trop. Dis. 4(2), e612 (2010).

13. Abidi, K., Dendane, T., Madani, N., Belayachi, J., Abouqal, R., \& Zeggwagh, A.A. The clinical picture of severe leptospirosis in critically ill patients. Care. Med .43(11), 1740-1741 (2017).

14. Luvira, V. et al. Etiologies of acute undifferentiated febrile illness in Bangkok, Thailand. J. Trop. Med. Hyg. 100(3), 622-629 (2019).

15. Wangdi, K., Kasturiaratchi, K., Nery, S.V., Lau, C.L., Gray, D.J., \& Clements, A.C. Diversity of infectious etiologies of acute undifferentiated febrile illnesses in South and Southeast Asia: a systematic review. BMC Infect. Dis. 19(1), 577 (2019).

16. Toyokawa, T., Ohnishi, M., \& Koizumi, N. Diagnosis of acute leptospirosis. Rev. Anti. Infect. Ther. 9(1), 111-121 (2011).

17. World Health Organization WHO, International Leptospirosis Society ILS. Human Leptospirosis: guidance for diagnosis, surveillance, and control. Preprint at https://apps.who.int/iris/handle/10665/42667 (2003).

18. Pappas, G., \& Cascio, Optimal treatment of leptospirosis querires and projections. Int. Antimicrob. Agents. 28(6), 491-496 (2006).

19. Smyth, L.D. et al. The microscopic agglutination test (MAT) is an unreliable predictor of infecting Leptospira serovart in Thailand. Am. J. Trop. Med. Hyg. 81(4), 695-697 (2009).

20. Niloofa, R. et al. Diagnosis of leptospirosis: comparison between Microscopic Agglutination Test, IgM-ELISA and IgM rapid immunochromatography test. PLoS One. 10(6), e0129236 (2015).

21. Doungchawee, G.et al. Development and evaluation of an immunochromatographic assay to detect serum anti-leptospiral lipopolysaccharide IgM in acute leptospirosis. Sci. Rep. 7(1), 2309 (2017).

22. Hunsperger, E.A. et al. Evaluation of commercially available diagnostic tests for the detection of dengue virus NS1 antigen and anti-Dengue virus IgM antibody. PLoS Negl. Trop. Dis. 8(10), e3171 (2014).

23. Gupta, N. et al. Scrub Typhus and Leptospirosis: the fallacy of diagnosing with IgM enzyme linked immunosorbant assay. J. Microb. Biochem. Technol. 8, 071-075 (2016).

24. Suttisunhakul, V. et al. Development of rapid enzyme-linked immunosorbent assays for detection of antibodies to Burkholderia pseudomallei. J. Clin. Microbiol. 54(5), 1259-1268 (2016).

25. Goris, M.G. et al. Towards the burden of human leptospirosis: duration of acute illness and occurrence of post-leptospirosis symptoms of patients in the Netherlands. PLoS One. 8(10), e76549 
(2013).

26. Esa, H.A.H., Amran, F., \& Halim, N.A.N. Evaluation of a commercial enzyme-linked immunosorbent assay kit for detection of IgM antibody against Leptospira in human sera for patients admitted in Malaysia hospitals. Microbiol. 7, 306 (2018).

27. Effler, P.V., Bogard,K., Domen, H.Y., Katz, A.R., Higa, H.Y., \& Sasaki, D.M. Evaluation of eight rapid screening tests for acute leptospirosis in Hawaii. J. Clin. Microbiol. 40(4), 1464-1469 (2002).

28. Blacksell, S.D. et al. Limited diagnostic capacities of two commercial assays for the detection of Leptospira immunoglobulin M antibodies in Laos. Vaccine. Immunol. 13(10), 1166-1169 (2006).

29. Wagenaar, J.F. et al. Rapid serological assays for leptospirosis are of limited value in southern Vietnam. Trop. Med. Parasitol. 98(8), 843-850 (2004).

30. Reller, M.E. et al. Leptospirosis as frequent cause of acute febrile illness in Southern Sri Lanka. Infect. Dis. 17(9), 1678-1684 (2011).

31. Courdurie, C. et al. Evaluation of different serological assays for early diagnosis of leptospirosis in Martinique (French West Indies). PloS Negl. Trop. Dis. 11(6), e0005678 (2017).

32. Desakorn, V. et al. Accuracy of commercial IgM ELISA for the diagnosis of human leptospirosis in Thailand. J. Trop. Med. Hyg. 86(3), 524-527 (2012).

33. Sakolvaree, Y. et al. Proteome and immunome of pathogenic Leptospira revealed by 2DE and 2DEimmunoblotting with immune serum. Asian. Pac. J. Allergy. Immunol. 25(1), 53-73 (2007).

34. Kositanont, U., Saetun, P., Krittanai, C., Doungchawee, G., Tribuddharat, C., \& Thongboonkerd, V. Application of immunoproteomic to leptospirosis: towards clinical diagnostics and vaccine discovery. Proteomics. Appl. 1(4), 400-409 (2007).

35. Lessa-Aquino, C. et al. Identification of seroreactive proteins of Leptospira interrogansserovar Copenhageni using a high-density protein microarray approach. PLoS Negl. Trop. Dis. 7(10), e2499 (2013).

36. Chalayon, P., Chanket, P., Boonchawalit, T., Chattanadee, S., Srimanote, P., \& Kalambaheti, T. Leptospirosis serodiagnosis by ELISA based on recombinant outer membrane protein. R. Soc. Trop. Med. Hyg. 105(5), 289-297 (2011).

37. Maneewatchararangsri, S. et al. Development of enzyme-linked immunosorbent assay for human leptospirosis serodiagnosis using Leptospira secretome antigen. Southeast Asian. J. Trop Med. Public. Health. 48(3), 576-584 (2017).

38. Doungchawee, G., Kositanont, U., Niwetpathomwat, A., Inwisai, T., Sagarasaeranee, P., \& Haake, D.A. Early diagnosis of leptospirosis by immunoglobulin $\mathrm{M}$ immunoblot testing. Clin. Vaccine. Immunol. 15(3), 492-498 (2008).

39. Madeira, F. et al. The EMBL-EBI search and sequence analysis tools APIs in 2019. Nucleic Acids Research. 47(W1), W636-W641 (2019).

40. Larsen, J.E., Lund, O., \& Neilsen, Improved method for predicting linear B-cell epitopes. Immunome. Res. 2, 2 (2006). 
41. Srimanote, P. et al. Recombinant ligA for leptospirosis diagnosis and ligA among the Leptospira clinical isolates. J. Microbiol. Method. 72(1), 73-81 (2008).

42. Alizadeh, S.A. et al. Diagnostic efficacy of Lsa63 antigen for human leptospirosis. Red. Crescent. Med. J. 16(3), e14753 (2014).

43. Natarajaseenivasan, K., Artiushin, S.C., Velineni, S., Vedhagiri, K., Vijayachari, P., \& Timoney, J.F. Surface-associated Hsp60 chaperonin of Leptospira interrogans serovar Autumnalis N2 strain as an immunoreactive protein. Clin. Microbiol. Infect. Dis. 30(11), 1383-1389 (2011).

44. Silva, M.V. et al. Behaviour of specific IgM, IgG and IgA class antibodies in human leptospirosis during the acute phase of the disease and during convalescence. J .Trop. Hyg. 98(4), 268-272 (1995).

45. Rosa, M.I. et al. IgM ELISA for leptospirosis diagnosis: a systematic review and meta-analysis. Saude. Colet. 22(12), 4001-4012 (2017).

46. Hinjoy, et al. Environmental and behavioral risk factors for severe leptospirosis in Thailand. Trop. Med. Infect. Dis. 4(2), 79 (2019).

47. Schreier, S., Doungchawee, G., Chadsuthi, S., Triampo, D. \& Triampo, W. Leptospirosis: current situation and trends of specific laboratory tests. Rev. Clin. Immunol. 9(3), 263-280 (2013).

48. Wuthiekanun, V. et al. Optimization of culture of Leptospira from humans with leptospirosis. Clin. Microbiol. 45(4), 1363-1365 (2007).

49. Chadsuthi, S. et al. Investigation on predominant Leptospira serovars and its distribution in humans and livestock in Thailand, 2010-2015. PloS Negl .Trop. Dis. 11(2), e0005228 (2017).

50. Thaipadungpanit, J. et al. Leptospiraspecies in floodwater during the 2011 floods in the Bangkok metropolitan region, Thailand. Am. J. Trop. Med. Hyg. 89(4), 794-796 (2013).

51. Cumberland, P.C., Everard, C.O.R. \& Levett, P. Assessment of the efficacy of the IgM enzyme-linked immunosorbent assay (ELISA) and microscopic agglutination test (MAT) in the diagnosis of acute leptospirosis. Am. J. Trop. Med. Hyg. 61(5), 731-734 (1996).

52. Sonthayanon, P. et al. Rapid diagnosis of scrub typhus in rural Thailand using polymerase chain reaction. Am. J. Trop. Med. Hyg. 75(6), 1099-1102 (2006).

53. Wongsrichanalai, C.et al. Co-infection with malaria and leptospirosis. Am. J. Trop. Med. Hyg. 68(5), $583-585$ (2003).

54. Chirathaworn, C., Inwattana, R, Pooworawan, Y., \& Suwancharoen, D. Interpretation of microscopic agglutination test for leptospirosis diagnosis and seroprevalence. Pac. J. Trop. Biomed. (Suppl1), S162-164 (2014).

\section{Tables}

Table 1. Diagnostic accuracy of $\mathrm{rGroEL}_{1-524}$ IgM-ELISA using culture and/or MAT as reference standard during the course of leptospirosis. 


\begin{tabular}{|llll|}
\hline Parameters & No./Total & IgM reactivity ${ }^{1}$ & Percentage (95\% Cl) \\
\hline \multicolumn{2}{|l}{ Sensitivity: Leptospirosis paired sera } & & \\
\hline Culture positive & $47 / 49$ & $0.37(0.06-0.92)$ & $95.9 \%(85.2-99.5)$ \\
\hline Single MAT ${ }^{3} 1: 400$ & $15 / 17$ & $0.47(0.13-0.78)$ & $88.2 \%(66.8-98.6)$ \\
\hline Seroconversion & $52 / 57$ & $0.45(0.13-1.01)$ & $91.2 \%(85.4-99.9)$ \\
\hline Specificity & & & \\
\hline Non-endemic healthy \& AUFI & $37 / 40$ & $0.03(0-0.22)$ & $92.5 \%(79.6-98.4)$ \\
\hline Other febrile illness sera & $39 / 54$ & $0.08(0-1.07)$ & $72.2 \%(58.4-83.5)$ \\
\hline Other febrile illness whole blood & $50 / 50$ & $0.02(0-0.10)$ & $100 \%(92.8-100)$ \\
\hline
\end{tabular}

Other infectious febrile illnesses sera consisted of melioidosis $(n=12)$, scrub typhus $(n=11)$, influenza $(n=11)$, malaria $(n=10)$, dengue fever $(n=10)$, and other febrile illness whole blood $(n=50)$

${ }^{1}$ Median AOD ELISA values (minimum-maximum values)

Table2. Diagnostic performances of commercial IgM-ELISA (Panbio) and IgG-ELISA (Virion-Serion) using optimized cut-offs and culture and/or MAT as reference standard during the course of leptospirosis.

\begin{tabular}{|c|c|c|c|c|}
\hline \multirow[t]{2}{*}{ Parameters $^{1}$} & \multicolumn{2}{|c|}{ Panbio IgM-ELISA ${ }^{2}$} & \multicolumn{2}{|c|}{ Virion-Serion IgG-ELISA ${ }^{3}$} \\
\hline & No./total & Percent (95\% Cl) & No./total & Percent (95\% Cl) \\
\hline \multicolumn{5}{|c|}{ Sensitivity: acute serum } \\
\hline Culture-positive & $15 / 30$ & $50.0 \%(31.3-68.7)$ & $4 / 30$ & $13.3 \%(3.8-30.7)$ \\
\hline MAT-negative & $24 / 38$ & $63.2 \%(45.9-78.2)$ & $4 / 38$ & $10.5 \%(2.9-24.8)$ \\
\hline DP01-3 & $17 / 31$ & $54.8 \%(36.0-72.7)$ & $3 / 31$ & $9.7 \%(2.0-25.8)$ \\
\hline DP01-10 & $31 / 52$ & $61.5 \%(47.0-74.7)$ & $9 / 51$ & $17.7 \%(8.4-30.9)$ \\
\hline \multicolumn{5}{|c|}{ Sensitivity: convalescent-serum } \\
\hline $\mathrm{DPO}^{3} 14$ & $30 / 42$ & $71.4 \%(55.4-84.3)$ & $23 / 47$ & $48.9 \%(34.1-63.9)$ \\
\hline \multicolumn{5}{|l|}{ Specificity } \\
\hline Controls & $84 / 97$ & $86.6 \%(78.2-92.7)$ & $98 / 131$ & $74.8 \%(66.5-84.9)$ \\
\hline
\end{tabular}

${ }^{1}$ The sensitivity of the ELISA tests was evaluated on culture-positive samples, MAT-negative $(<1: 50)$ of seroconversion MAT titer paired sera. Sensitivity was also determined during the course of illness from 
DP01-3, DP01-10 acute-sera, and ${ }^{3}$ DP014 of convalescent sera. Specificity was determined from all control samples, including healthy, AUFI, and other febrile illnesses in leptospirosis-endemic areas.

2 Optimized cut-off for Panbio IgM-ELISA was ${ }^{37}$ panbiounits. ${ }^{3}$ Optimized cut-off for Virion-Serion IgGELISA was ${ }^{30} 0.35$ OD ELISA.

\section{Figures}

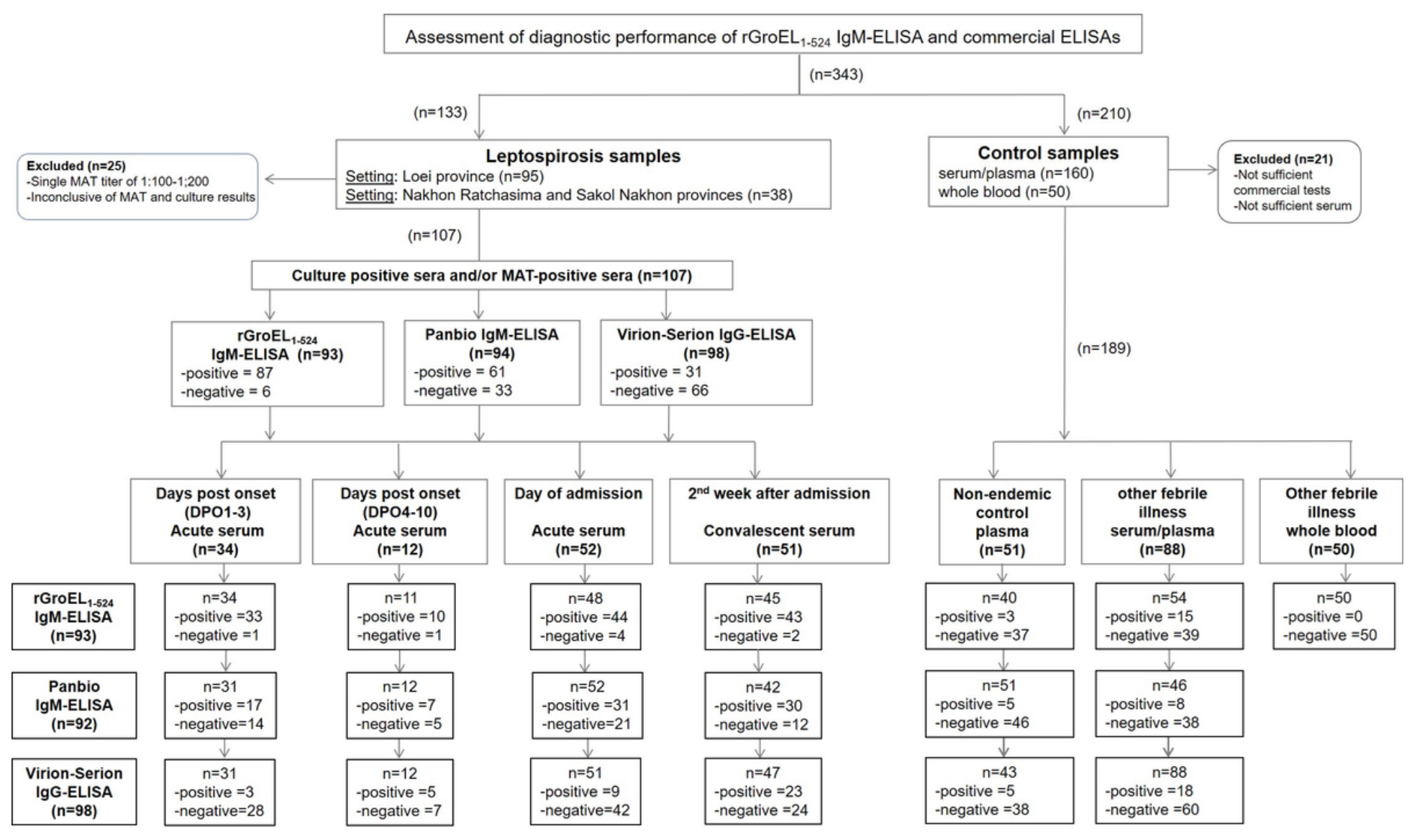

Figure 1

Flow diagram of the assessment of diagnostic accuracy of rGroEL1-524 IgM-ELISA, and commercial ELISAs. A culture and/or MAT-positive leptospirosis paired sera $(n=107)$ and control samples $(n=189)$ consisting of 51 non-endemic control plasma (seronegative and negative for leptospirosis lgM detection), 88 serum/plasma and 50 whole blood of laboratory-confirmed infectious diseases other than leptospirosis were subjected to an assessment of the diagnostic sensitivity and specificity of the following tests: (i) rGroEL1-524 IgM-ELISA, and commercial (ii) Panbio Leptospira IgM-ELISA, and (iii) Serion-Virion classic Leptospira IgG-ELISA and compared to reference MAT and culture methods. Confirmed leptospirosis sera were acute sera collected on the date of admission $(n=52)$ and convalescent-sera collected in the later 2 weeks $(n=51)$. Acute-phase sera were classified according to days post-onset of symptoms (DPO) into DP01-3 ( $n=34)$, and DPO4-10 ( $n=12)$ acute serum. Control samples $(n=189)$ were grouped into non-endemic healthy and AUFI control plasma $(n=51)$, and other 
febrile illness serum/plasma $(n=88)$ and other febrile illness whole blood $(n=50)$. Of 343 sera, 25 samples (MAT=1:100-200) and 21 control sera were excluded from study. Positive results of the commercial ELISAs were considered using the recommended cut-offs.
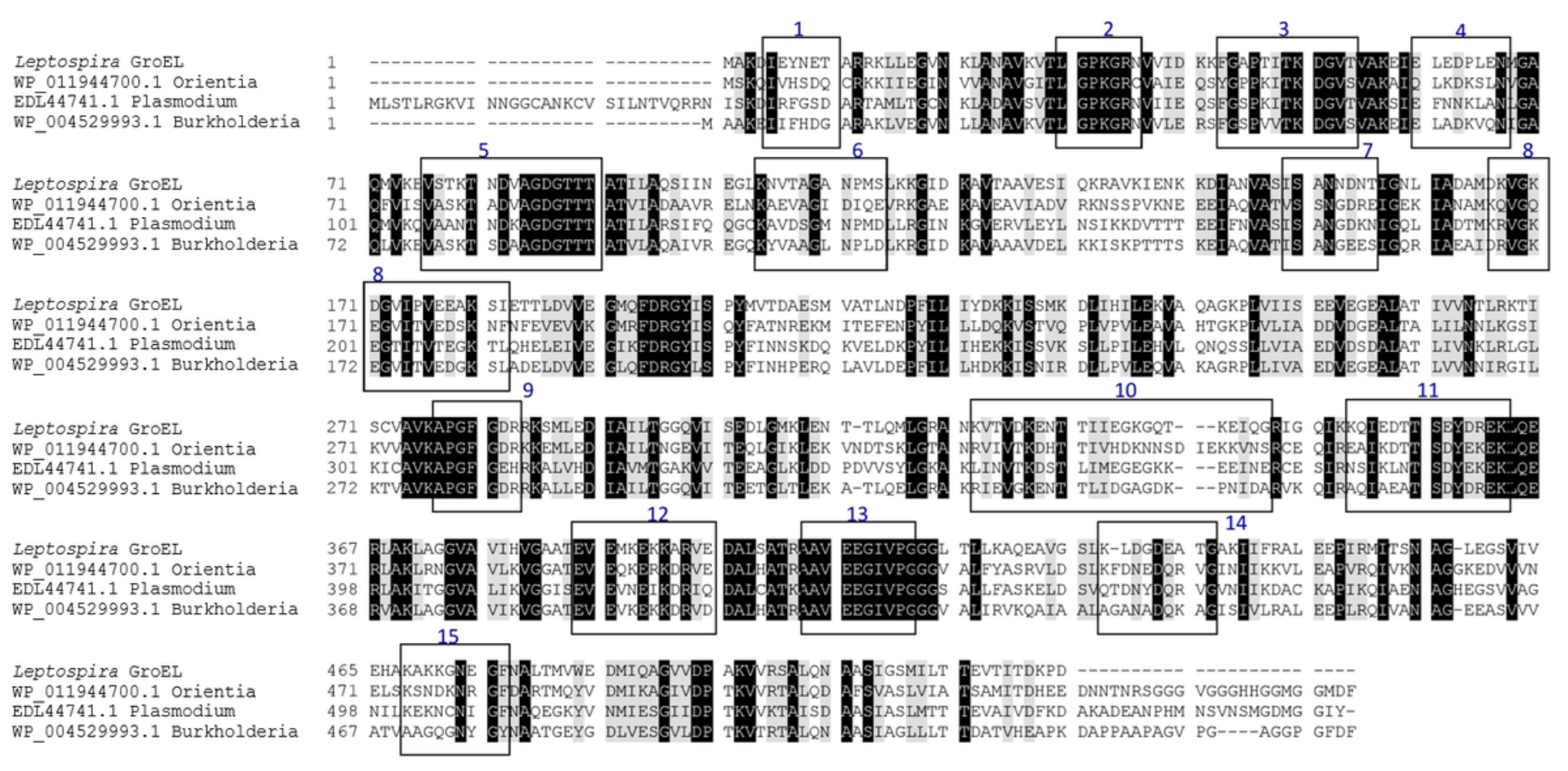

Figure 2

Multiple sequence alignment of orthologous GroEL proteins, and prediction of linear B-epitope peptides. GroEL sequence of L. interrogans serovar Icterohaemorrhagiae, and multispecies Burkholderia (WP_004529993.1), Plasmodium vivax (EDL44741.1), and Orientia tsutsugamushi (WP_011944700.1), were multiply aligned using Clustal Omega multiple sequence alignment program, and analyzed with the BioEdit sequence alignment editor. The GroEL sequence of $L$. interrogans serovar Icterohemorrhagiae was subjected to Bepipred-1.0 Linear Epitope Prediction. Epitopes with a Bepipred score of more than 0.35 were predicted to be highly immunogenic epitopes. There are 15 predicted epitope peptides with $100 \%$ consensus residues-i.e., \#1:5-IEYNET-10 (16.6\%), \#2:30-LGPKGRN-36 (85.7\%), \#3:43-FGAPTITKDGVT-54 (58.3\%), \#4:60-ELEDPLEN-67 (12.5\%), \#5:76-VSTKTNDVAGDGTTT-90 (66.6\%), \#6:104-KNVTAGANPMS114 (18.1\%), \#7:149-ISANNDNT-156 (25\%), \#8:166-DKVGKDGVIPVEEAKSI-182 (35.2\%), \#9:277APGFGDR-283 (71.4\%), \#10:321-KVTVDKENTTIIEGKGQTKEIQG-343 (13.0\%), \#11:350KQIEDTTSEYDREK-363 (42.8\%), \#12:385-EVEMKEKKARVE-396 (50\%), 13:404-AAVEEGIVPG-413 (100\%), \#14:429-KLDGDEATG-437 (22.2\%), and \#15:468-KAKKGNEGF-476 (22.2\%). Consensus residues are highlighted. Black-shaded residues represent $100 \%$ identity. Grey-shaded residues represent $50 \%$ similarity. Dash (-) represents gap. 
A

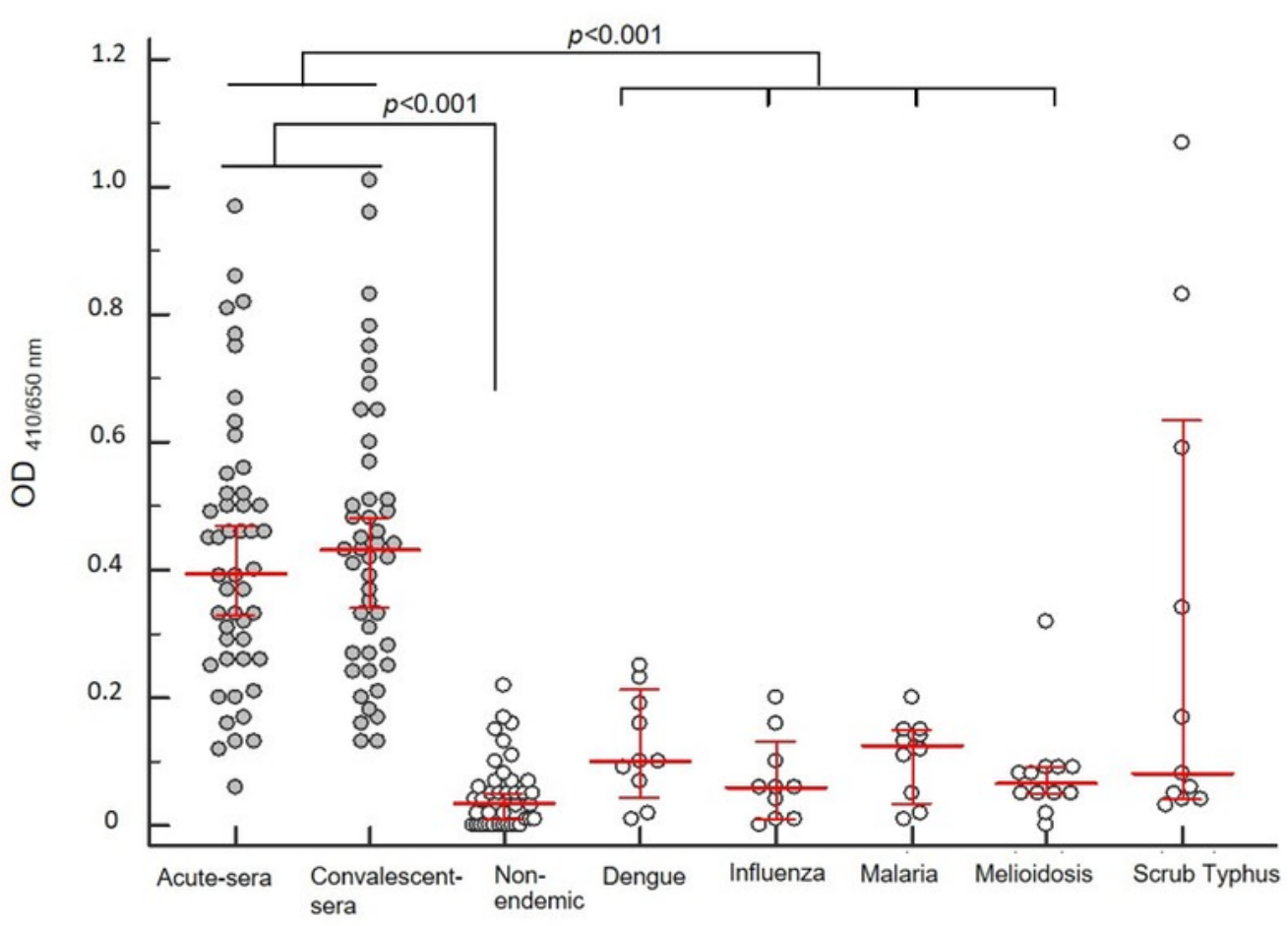

B

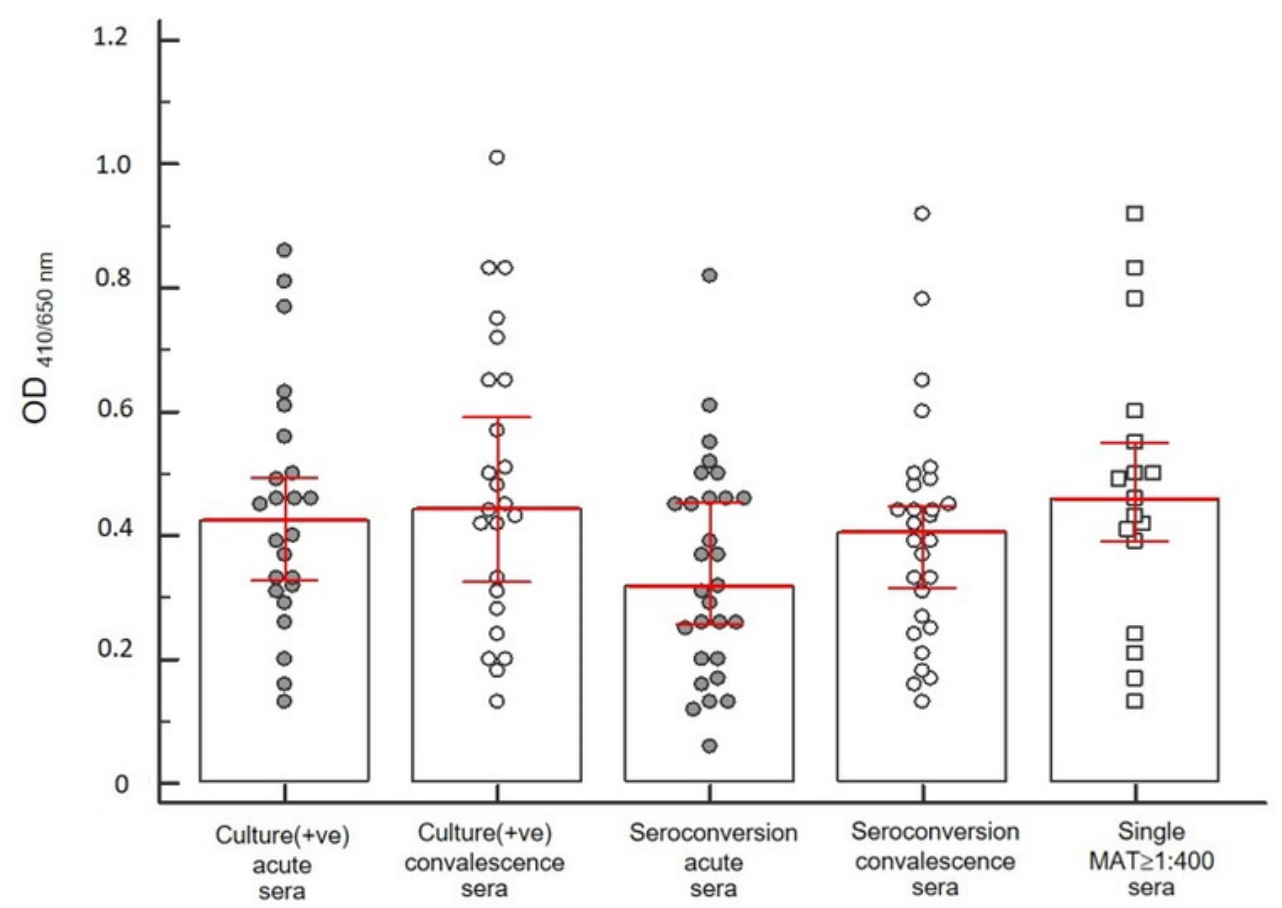

Figure 3

IgM reactivity of confirmed leptospirosis paired sera and controls assessed by rGroEL1-524 IgM-ELISA. (A) The IgM reactivity of acute and convalescent leptospirosis sera (•), non-endemic healthy and AUFI control plasma (o), and other febrile illness control samples (o), comprising dengue fever, influenza, malaria, melioidosis and scrub typhus subgroups, were assessed rGroEL1-524 IgM-ELISA assay. (B) IgM reactivity of confirmed leptospirosis acute $(\cdot)$ and convalescent (o) sera were classified according to gold- 
standard methods including culture-positive and MAT-positive subgroups by seroconversion MAT titers and single MAT>1:400 (0) samples. Individual IgM reactivity is expressed as AOD ELISA. The median AOD ELISA value and standard deviation (SD) of each subgroup are indicated. $p<0.001$ is considered significantly different.

\section{Supplementary Files}

This is a list of supplementary files associated with this preprint. Click to download.

- Tableofthemanuscript.doc

- Supplementarylnformation.doc 